\title{
Approximation of Integrable Functions by Wavelet Expansions
}

\author{
Włodzimierz Łenski and Bogdan Szal
}

\begin{abstract}
Walter (J Approx Theory 80:108-118, 1995), Xiehua (Approx Theory Appl 14(1):81-90, 1998) and Lal and Kumar (Lobachevskii J Math 34(2):163-172, 2013) established results on pointwise and uniform convergence of wavelet expansions. Working in this direction new more general theorems on degree of pointwise approximation by such expansions have been proved.
\end{abstract}

Mathematics Subject Classification. 42C40, 41A25.

Keywords. pointwise approximation, wavelet expansions.

\section{Introduction}

In this paper we use the following notations.

$L^{p}(\mathbb{R})(1<p<\infty)$ denotes the space of measurable, integrable with $p-t h$ power functions $f$. The norm of $f \in L^{p}(\mathbb{R})$ is written by $\|f\|_{L^{p}(\mathbb{R})}$.

$l^{2}(\mathbb{Z})$ is the vector space of square-summable functions.

A multiresolution approximation of $L^{2}(\mathbb{R})$ (see [3]) is a sequence $\left(V_{j}\right)_{j \in Z}$ of closed subspaces of $L^{2}(\mathbb{R})$ such that the following hold:

$$
\begin{aligned}
& \forall_{j \in \mathbb{Z}} V_{j} \subset V_{j+1}, \\
& \bigcup_{j \in \mathbb{Z}} V_{j} \text { is dense in } L^{2}(\mathbb{R}) \text { and } \bigcap_{j \in \mathbb{Z}} V_{j}=\varnothing, \\
& \forall_{j \in \mathbb{Z}} f(x) \in V_{j} \Leftrightarrow f(2 x) \in V_{j+1}, \\
& \forall_{j, k \in \mathbb{Z}} f(x) \in V_{j} \Leftrightarrow f\left(x-2^{-j} k\right) \in V_{j},
\end{aligned}
$$

There exists an isomorphism $I$ from $V_{0}$ onto $l^{2}(\mathbb{Z})$ which commutes with the action of $\mathbb{Z}$.

We consider orthonormal bases of wavelets in $L^{2}(\mathbb{R})($ see $[1])$. These are functions of the form

$$
\psi_{j, k}(x)=2^{\frac{j}{2}} \psi\left(2^{j} x-k\right),
$$


for some fixed function $\psi$ and are in turn based on a scaling function $\varphi$. In addition to the ladder of approximation spaces

$$
\cdots \subset V_{-1} \subset V_{0} \subset V_{1} \subset \cdots \subset L^{2}(\mathbb{R})
$$

we have the orthogonal complement of each in the next higher one on the ladders i.e.,

$$
\cdots V_{0} \oplus W_{0}=V_{1}, V_{1} \oplus W_{1}=V_{2} \cdots
$$

The translates of the scaling function $\varphi$ are an orthonormal basis of $V_{0}$ while the translates of $\psi$ are an orthogonal basis of $W_{o}$ (see [1]). The same holds for their dilations in $V_{j}$ and $W_{j}$. Hence each $f \in L^{2}(\mathbb{R})$ has a representation

$$
f(x)=f_{n}(x)+f_{n}^{\perp}(x),
$$

where

$$
f_{n}(x)=\sum_{k=-\infty}^{\infty} a_{n, k} \varphi_{n, k}(x), \quad \varphi_{n, k}(x)=2^{\frac{n}{2}} \varphi\left(2^{n} x-k\right)
$$

and

$$
f_{n}^{\perp}(x)=\sum_{j=-n}^{\infty} \sum_{k=-\infty}^{\infty} b_{j, k} \psi_{j, k}(x), \quad \psi_{j, k}(x)=2^{\frac{j}{2}} \psi\left(2^{j} x-k\right)
$$

and

$$
f(x)=\sum_{j=-\infty}^{\infty} \sum_{k=-\infty}^{\infty} b_{j, k} \psi_{j, k}(x), \quad \text { with } \quad f_{n}(x)=\sum_{j=-\infty}^{n} \sum_{k=-\infty}^{\infty} b_{j, k} \psi_{j, k}(x),
$$

where $a_{n, k}, b_{j, k}$ are expansion coefficients of $f . f_{n}$ is said to be the partial sums of the wavelet expansion. It is given

$$
f_{n}(x)=\int_{-\infty}^{\infty} q_{n}(x, t) f(t) d t=\int_{-\infty}^{\infty} p_{n}(x, t) f(t) d t,
$$

by the reproducing kernels

$$
q_{n}(x, t)=\sum_{k=-\infty}^{\infty} \varphi_{n, k}(x) \varphi_{n, k}(t) \text { and } p_{n}(x, t)=\sum_{j=-\infty}^{n} \sum_{k=-\infty}^{\infty} \psi_{j, k}(x) \psi_{j, k}(t)
$$

(see [4]).

The pointwise modules of continuity of the function $f$ at point $x$ are given by

$$
\begin{gathered}
\omega_{x}(f, \delta)=\sup _{|t| \leq \delta}|f(t+x)-f(x)|, \\
w_{x}(f, \delta)=\frac{1}{\delta} \int_{-\delta}^{\delta}|f(t+x)-f(x)| d t,
\end{gathered}
$$

for fixed $x$.

The deviation $f_{n}(x)-f(x)$ was estimated by Xiehua [11, Theorem 3.1, p. 84] ( see also [5, Theorem 3.1] and [10]) as follows: 
Theorem A. Assume the scaling function $\varphi$ satisfies the condition

$$
|\varphi(x)| \leq \frac{C}{1+|x|^{\alpha}}, \quad \alpha>1 .
$$

If $f \in L^{2}(\mathbb{R})$ is continuous at $x$, then we have

$$
\begin{aligned}
& \left|f_{n}(x)-f(x)\right| \\
& \quad=O(1)\left\{\left(\|f\|_{L^{2}(\mathbb{R})}+\frac{1}{\alpha-1}|f(x)|\right) 2^{-(\alpha-1) n}+\sum_{k=1}^{2^{n}} k^{-\alpha} \omega_{x}\left(f, k 2^{-n}\right)\right\},
\end{aligned}
$$

where "O" depends only on $C$.

We also note the following well known result of Daubechies.

Theorem B [2, Theorem 9.1.6.]. Let $\varphi^{\prime}$ be a continuous function such that $\varphi$ and $\varphi^{\prime}$ satisfy $(1)$. If $\varphi_{n, k}$ constitute an orthonormal basis on $L^{2}(\mathbb{R})$, then the $\left\{\varphi_{n, k}: n, k \in \mathbb{Z}\right\}$ also constitute an unconditional basis for all the spaces $L^{p}(\mathbb{R})$ $(1<p<\infty)$.

Here, we will give more general and precise estimates of the pointwise convergence of wavelet expansions.

\section{Statement of the Results}

We determine the degree of approximation of functions by wavelet expansions.

Theorem 1. Assume that a continuous function $\varphi^{\prime}$ is such that $\varphi$ and $\varphi^{\prime}$ satisfy (1). If $f \in L^{p}(\mathbb{R})(1<p<\infty)$, then

$$
\left|f_{n}(x)-f(x)\right| \leq K\left\{2 w_{x}\left(f, 2^{-n}\right)+\alpha 2^{n(1-\alpha)} \int_{2^{-n}}^{\infty} t^{-\alpha} w_{x}(f, t) d t\right\}
$$

at every point $x$ and with a positive constant $K$ dependent on $C$.

Proof. From (1) it is easy to say that

$$
|q(x, t)| \leq \frac{K}{1+|x-t|^{\alpha}} .
$$

Following Walter [10], Meyer [6] and Novikov, Protasov, Skopina [7]

$$
\int_{-\infty}^{\infty} q_{n}(x, t) d t=1, \quad \text { for } x \in \mathbb{R}
$$

and we have

$f_{n}(x)-f(x)=\int_{-\infty}^{\infty} q_{n}(x, t)\{f(t)-f(x)\} d t=\int_{-\infty}^{x-2^{-n}}+\int_{x-2^{-n}}^{x+2^{-n}}+\int_{x+2^{-n}}^{\infty}$.

Next, using (2) 


$$
\begin{aligned}
\left|\int_{x-2^{-n}}^{x+2^{-n}}\right| \leq & \int_{x-2^{-n}}^{x+2^{-n}} \frac{K 2^{n}}{1+\left(2^{n}|x-t|\right)^{\alpha}}|f(t)-f(x)| d t \\
= & \int_{0}^{2^{-n}} \frac{K 2^{n}}{1+\left(2^{n}|t|\right)^{\alpha}}[|f(t+x)-f(x)|+|f(-t+x)-f(x)|] d t \\
= & \int_{0}^{2^{-n}} \frac{K 2^{n}}{1+\left(2^{n}|t|\right)^{\alpha}}\left[\frac{d}{d t} \int_{-t}^{t}|f(u+x)-f(x)| d u\right] d t \\
= & {\left[\frac{K 2^{n}}{1+\left(2^{n}|t|\right)^{\alpha}} \int_{-t}^{t}|f(u+x)-f(x)| d u\right]_{0}^{2^{-n}} } \\
& +\int_{0}^{2^{-n}} \frac{\alpha K 2^{n(1+\alpha)}|t|^{\alpha-1}}{\left[1+\left(2^{n}|t|\right)^{\alpha}\right]^{2}}\left[\int_{-t}^{t}|f(u+x)-f(x)| d u\right] d t \\
= & \frac{K}{1+1^{\alpha}} w_{x}\left(f, 2^{-n}\right)+w_{x}\left(f, 2^{-n}\right) \int_{0}^{2^{-n}} \frac{\alpha K 2^{n \alpha} t^{\alpha-1}}{\left[1+\left(2^{n} t\right)^{\alpha}\right]^{2}} d t \\
= & K w_{x}\left(f, 2^{-n}\right) .
\end{aligned}
$$

and

$$
\begin{aligned}
& \left|\int_{-\infty}^{x-2^{-n}}+\int_{x+2^{-n}}^{\infty}\right| \leq\left(\int_{-\infty}^{x-2^{-n}}+\int_{x+2^{-n}}^{\infty}\right) \frac{K 2^{n}}{1+\left(2^{n}|x-t|\right)^{\alpha}}|f(t)-f(x)| d t \\
& \leq\left(\int_{-\infty}^{-2^{-n}}+\int_{2^{-n}}^{\infty}\right) \frac{K 2^{n}}{1+\left(2^{n}|t|\right)^{\alpha}}|f(t+x)-f(x)| d t \\
& \leq K 2^{n(1-\alpha)}\left(\int_{-\infty}^{-2^{-n}}+\int_{2^{-n}}^{\infty}\right) \frac{|f(t+x)-f(x)|}{|t|^{\alpha}} d t \\
& =K 2^{n(1-\alpha)} \int_{2^{-n}}^{\infty} t^{-\alpha}\left[\frac{d}{d t} \int_{-t}^{t}|f(u+x)-f(x)| d u\right] d t \\
& =K 2^{n(1-\alpha)}\left[t^{-\alpha} \int_{-t}^{t}|f(u+x)-f(x)| d u\right]_{2^{-n}}^{\infty} \\
& +\alpha K 2^{n(1-\alpha)} \int_{2^{-n}}^{\infty} t^{-\alpha-1}\left[\int_{-t}^{t}|f(u+x)-f(x)| d u\right] d t .
\end{aligned}
$$

Observing that

$$
\begin{aligned}
& \lim _{t \rightarrow \infty} t^{-\alpha} \int_{-t}^{t}|f(u+x)-f(x)| d u \\
& \leq \lim _{t \rightarrow \infty}\left\{2^{1-\frac{1}{p}} t^{-\alpha+1}\left[\frac{1}{t} \int_{-t}^{t}|f(u+x)|^{p} d u\right]^{1 / p}+2 t^{-\alpha+1}|f(x)|\right\} \\
& \quad \leq \lim _{t \rightarrow \infty}\left\{2^{1-\frac{1}{p}} t^{-\alpha+1-\frac{1}{p}}\|f\|_{L^{p}(\mathbb{R})}+2 t^{-\alpha+1}|f(x)|\right\}=0
\end{aligned}
$$


we obtain

$$
\left|\int_{-\infty}^{x-2^{-n}}+\int_{x+2^{-n}}^{\infty}\right| \leq K w_{x}\left(f, 2^{-n}\right)+K 2^{n(1-\alpha)} \alpha \int_{2^{-n}}^{\infty} t^{-\alpha} w_{x}(f, t) d t .
$$

Collecting our partial estimates the result follows.

Corollary 1. If $f \in\left\{g \in L^{p}(\mathbb{R}): w_{x}(g, \delta)=O\left(w_{x}(\delta)\right)\right\}$ where $w_{x}$ is a function of modulus of continuity type, then by the monotonicity of $\frac{w_{x}(\delta)}{\delta}$ and $\alpha>2$, we obtain

$$
\left|f_{n}(x)-f(x)\right|=O\left(w_{x}\left(2^{-n}\right)\right) .
$$

Theorem 2. Under the assumptions of Theorem 1, we have

$$
\begin{aligned}
\left|f_{n}(x)-f(x)\right| \leq & 2 K w_{x}\left(f, 2^{-n}\right)+K \alpha 2^{n(1-\alpha)} \int_{2^{-n}}^{1} t^{-\alpha} w_{x}(f, t) d t \\
& +K 2^{n(1-\alpha)}\left[\left(\frac{2}{(\alpha q-1)^{1 / q}}+2^{\frac{1}{q}}\right)\|f\|_{L^{p}(\mathbb{R})}\right. \\
& \left.+2\left(\frac{1}{\alpha-1}+1\right)|f(x)|\right],
\end{aligned}
$$

where $\frac{1}{p}+\frac{1}{q}=1$.

Proof. Similarly as above

$f_{n}(x)-f(x)=\int_{-\infty}^{\infty} q_{n}(x, t)\{f(t)-f(x)\} d t=\int_{-\infty}^{x-2^{-n}}+\int_{x-2^{-n}}^{x+2^{-n}}+\int_{x+2^{-n}}^{\infty}$ and

$$
\left|\int_{x-2^{-n}}^{x+2^{-n}}\right| \leq K w_{x}\left(f, 2^{-n}\right)
$$

Further

$$
\begin{aligned}
& \left|\int_{-\infty}^{x-2^{-n}}+\int_{x+2^{-n}}^{\infty}\right| \\
& \quad \leq K 2^{n(1-\alpha)} \int_{2^{-n}}^{\infty} \frac{|f(t+x)-f(x)|+|f(-t+x)-f(x)|}{t^{\alpha}} d t \leq \int_{2^{-n}}^{1}+\int_{1}^{\infty}
\end{aligned}
$$

and

$$
\begin{aligned}
\int_{2^{-n}}^{1}= & K 2^{n(1-\alpha)} \int_{2^{-n}}^{1} t^{-\alpha}\left[\frac{d}{d t} \int_{-t}^{t}|f(u+x)-f(x)| d u\right] d t \\
= & K 2^{n(1-\alpha)}\left[t^{-\alpha} \int_{-t}^{t}|f(u+x)-f(x)| d u\right]_{2^{-n}}^{1} \\
& +\alpha K 2^{n(1-\alpha)} \int_{2^{-n}}^{1} t^{-\alpha-1}\left[\int_{-t}^{t}|f(u+x)-f(x)| d u\right] d t
\end{aligned}
$$




$$
\begin{aligned}
\leq & K 2^{n(1-\alpha)}\left[2^{1-\frac{1}{p}}\|f\|_{L^{p}(\mathbb{R})}+2|f(x)|\right]+K w_{x}\left(f, 2^{-n}\right) \\
& +K 2^{n(1-\alpha)} \alpha \int_{2^{-n}}^{1} t^{-\alpha} w_{x}(f, t) d t \\
\int_{1}^{\infty} \leq & K 2^{n(1-\alpha)}\left\{\left(\int_{1}^{\infty} t^{-q \alpha} d t\right)^{\frac{1}{q}}\left(\int_{\mathbb{R}}|f(t+x)|^{p} d t\right)^{\frac{1}{p}}\right. \\
& \left.+\left(\int_{1}^{\infty} t^{-q \alpha} d t\right)^{\frac{1}{q}}\left(\int_{\mathbb{R}}|f(-t+x)|^{p} d t\right)^{\frac{1}{p}}+2|f(x)| \int_{1}^{\infty} t^{-\alpha} d t\right\} \\
\leq & K 2^{n(1-\alpha)}\left(\frac{2}{(\alpha q-1)^{1 / q}}\|f\|_{L^{p}(\mathbb{R})}+\frac{2|f(x)|}{\alpha-1}\right) .
\end{aligned}
$$

Thus we obtain the desired estimate.

Corollary 2. Under the assumptions of Theorem 1 and if $w_{x}(f, \delta)=O\left(\delta^{\gamma}\right)$, then by Theorem 2

$$
\left|f_{n}(x)-f(x)\right|=\left\{\begin{array}{l}
O\left(2^{-n \gamma}\right) \quad \text { when } 0<\gamma<\alpha-1 \\
O\left(2^{-n(\alpha-1)}\right) \quad \text { when } \gamma>\alpha-1 \\
O\left(n 2^{-n(\alpha-1)}\right) \quad \text { when } \gamma=\alpha-1
\end{array}\right.
$$

The same order of approximation one can find in the paper of Skopina $[9]$.

Remark 1. If $x$ is a point of continuity of $f \in L^{p}(\mathbb{R})$, then $\omega_{x}(f, \delta)=o(1)$, whence $w_{x}(f, \delta)=o(1)$ and thus

$$
\left|f_{n}(x)-f(x)\right|=o(1) .
$$

We also show our estimates in the following summation forms.

Theorem 3. Under the assumptions of Theorem 1

$$
\begin{aligned}
\left|f_{n}(x)-f(x)\right| \leq & K_{\alpha} 2^{n(1-\alpha)} \sum_{\nu=0}^{2^{n}-2}(\nu+2)^{\alpha-2} w_{x}\left(f, \frac{1}{\nu+1}\right) \\
& +2 \alpha K \sum_{\nu=2^{n}}^{\infty} \nu^{-\alpha} w_{x}\left(f, \frac{\nu+1}{2^{n}}\right)
\end{aligned}
$$

where $K_{\alpha}=K\left[2^{\alpha+2}+2 \alpha\right]$.

Proof. Since $\delta w_{x}(f, \delta)$ is nondecreasing function of $\delta$, therefore for $N \geq 1$

$$
\begin{aligned}
& \int_{2^{-n}}^{N} t^{-\alpha} w_{x}(f, t) d t=\left(\int_{2^{-n}}^{1}+\int_{1}^{N}\right) t^{-\alpha} w_{x}(f, t) d t \\
& \quad=\int_{1}^{2^{n}} u^{\alpha-1} \frac{1}{u} w_{x}\left(f, \frac{1}{u}\right) d u+\sum_{\nu=2^{n}}^{N 2^{n}-1} \int_{\nu 2^{-n}}^{(\nu+1) 2^{-n}} t^{-\alpha-1} t w_{x}(f, t) d t
\end{aligned}
$$




$$
\begin{aligned}
& =\sum_{\nu=1}^{2^{n}-1} \int_{\nu}^{\nu+1} u^{\alpha-1} \frac{1}{u} w_{x}\left(f, \frac{1}{u}\right) d u+\sum_{\nu=2^{n}}^{N 2^{n}-1} \int_{\nu 2^{-n}}^{(\nu+1) 2^{-n}} t^{-\alpha-1} t w_{x}(f, t) d t \\
& \leq \sum_{\nu=1}^{2^{n}-1} \frac{1}{\nu} w_{x}\left(f, \frac{1}{\nu}\right)\left[\frac{u^{\alpha}}{\alpha}\right]_{u=\nu}^{\nu+1}+\sum_{\nu=2^{n}}^{N 2^{n}-1} \frac{\nu+1}{2^{n}} w_{x}\left(f, \frac{\nu+1}{2^{n}}\right)\left[\frac{t^{-\alpha}}{-\alpha}\right]_{t=\nu 2^{-n}}^{(\nu+1) 2^{-n}} \\
& \leq \sum_{\nu=1}^{2^{n}-1} \frac{1}{\nu} w_{x}\left(f, \frac{1}{\nu}\right)(\nu+1)^{\alpha-1}+\sum_{\nu=2^{n}}^{N 2^{n}-1} \frac{\nu+1}{2^{n}} w_{x}\left(f, \frac{\nu+1}{2^{n}}\right) \frac{\nu^{-\alpha-1}}{2^{-\alpha n}} \\
& \leq 2 \sum_{\nu=1}^{2^{n}-1} w_{x}\left(f, \frac{1}{\nu}\right)(\nu+1)^{\alpha-2}+2^{n(\alpha-1)+1} \sum_{\nu=2^{n}}^{N 2^{n}-1} \nu^{-\alpha} w_{x}\left(f, \frac{\nu+1}{2^{n}}\right) \\
& \leq 2 \sum_{\nu=0}^{2^{n}-2}(\nu+2)^{\alpha-2} w_{x}\left(f, \frac{1}{\nu+1}\right)+2^{n(\alpha-1)+1} \sum_{\nu=2^{n}}^{\nu^{-\alpha}} w_{x}\left(f, \frac{\nu+1}{2^{n}}\right) \\
& \leq 2 \sum_{\nu=0}^{2^{n}-2}(\nu+2)^{\alpha-2} w_{x}\left(f, \frac{1}{\nu+1}\right)+2^{n(\alpha-1)+1} \sum_{\nu=2^{n}}^{\infty} \nu^{-\alpha} w_{x}\left(f, \frac{\nu+1}{2^{n}}\right)
\end{aligned}
$$

and

$$
\begin{aligned}
& \sum_{\nu=0}^{2^{n}-2}(\nu+2)^{\alpha-2} w_{x}\left(f, \frac{1}{\nu+1}\right) \\
& \quad \geq \sum_{\nu=2^{n-1}-1}^{2^{n}-2}(\nu+2)^{\alpha-1} \frac{1}{\nu+2} w_{x}\left(f, \frac{1}{\nu+1}\right) \\
& \quad \geq \frac{1}{2} \sum_{\nu=2^{n-1}-1}^{2^{n}-2}(\nu+2)^{\alpha-1} \frac{1}{\nu+1} w_{x}\left(f, \frac{1}{\nu+1}\right) \\
& \quad \geq \frac{1}{2} \sum_{\nu=2^{n-1}-1}^{2^{n}-2}(\nu+2)^{\alpha-1} \frac{1}{2^{n}} w_{x}\left(f, \frac{1}{2^{n}}\right) \geq \frac{1}{2} \frac{1}{2^{n}} w_{x}\left(f, \frac{1}{2^{n}}\right)\left(2^{n-1}+1\right)^{\alpha-1} 2^{n-1} \\
& \geq \frac{1}{2} \frac{1}{2^{n}} w_{x}\left(f, \frac{1}{2^{n}}\right) 2^{(n-1) \alpha}=\frac{1}{2^{\alpha+1}} 2^{n(\alpha-1)} w_{x}\left(f, \frac{1}{2^{n}}\right) .
\end{aligned}
$$

Thus, by Theorem 1 our result follows.

Theorem 4. Under the assumptions of Theorem 1

$$
\begin{aligned}
\left|f_{n}(x)-f(x)\right| \leq & K_{\alpha} \sum_{\nu=1}^{2^{n}-1} \nu^{-\alpha} w_{x}\left(f, \frac{\nu+1}{2^{n}}\right) \\
& +K 2^{n(1-\alpha)}\left[\left(\frac{2}{(\alpha q-1)^{1 / q}}+2^{\frac{1}{q}}\right)\|f\|_{L^{p}(\mathbb{R})}\right. \\
& \left.+2\left(\frac{1}{\alpha-1}+1\right)|f(x)|\right],
\end{aligned}
$$

where $K_{\alpha}=K\left[2^{\alpha+2}+2 \alpha\right]$. 
Proof. Using the monotonicity of $\delta w_{x}(f, \delta)$ we obtain that

$$
\begin{aligned}
\int_{2^{-n}}^{1} t^{-\alpha} w_{x}(f, t) d t & =\sum_{\nu=1}^{2^{n}-1} \int_{\nu 2^{-n}}^{(\nu+1) 2^{-n}} t^{-\alpha-1} t w_{x}(f, t) d t \\
& \leq \sum_{\nu=1}^{2^{n}-1} \frac{\nu+1}{2^{n}} w_{x}\left(f, \frac{\nu+1}{2^{n}}\right) \int_{\nu 2^{-n}}^{(\nu+1) 2^{-n}} t^{-\alpha-1} d t \\
& =\sum_{\nu=1}^{2^{n}-1} \frac{\nu+1}{2^{n}} w_{x}\left(f, \frac{\nu+1}{2^{n}}\right)\left[\frac{t^{-\alpha}}{-\alpha}\right]_{t=\nu 2^{-n}}^{(\nu+1) 2^{-n}} \\
& =\frac{1}{-\alpha} \sum_{\nu=1}^{2^{n}-1} \frac{\nu+1}{2^{n}} w_{x}\left(f, \frac{\nu+1}{2^{n}}\right)\left[\left(\frac{2^{n}}{\nu+1}\right)^{\alpha}-\left(\frac{2^{n}}{\nu}\right)^{\alpha}\right] \\
& =\frac{1}{\alpha} \sum_{\nu=1}^{2^{n}-1} \frac{\nu+1}{2^{(1-\alpha) n}} w_{x}\left(f, \frac{\nu+1}{2^{n}}\right)\left[\nu^{-\alpha}-(\nu+1)^{-\alpha}\right] \\
& \leq 2^{(\alpha-1) n} \sum_{\nu=1}^{2^{n}-1}(\nu+1) \nu^{-\alpha-1} w_{x}\left(f, \frac{\nu+1}{2^{n}}\right) \\
& \leq 2^{(\alpha-1) n+1} \sum_{\nu=1}^{2^{n}-1} \nu^{-\alpha} w_{x}\left(f, \frac{\nu+1}{2^{n}}\right)
\end{aligned}
$$

and

$$
\begin{aligned}
& \sum_{\nu=1}^{2^{n}-1} \nu^{-\alpha} w_{x}\left(f, \frac{\nu+1}{2^{n}}\right) \\
& \quad \geq 2^{n} \sum_{\nu=1}^{2^{n}-1}(\nu+1)^{-1-\alpha} \frac{\nu+1}{2^{n}} w_{x}\left(f, \frac{\nu+1}{2^{n}}\right) \geq w_{x}\left(f, \frac{1}{2^{n}}\right) \sum_{\nu=1}^{2^{n}-1}(\nu+1)^{-1-\alpha} \\
& \quad \geq(1+1)^{-1-\alpha} w_{x}\left(f, \frac{1}{2^{n}}\right)=2^{-1-\alpha} w_{x}\left(f, \frac{1}{2^{n}}\right) .
\end{aligned}
$$

Hence, by Theorem 2, analogously as above, our result follows.

Remark 2. Since

$$
w_{x}(f, \delta) \leq 2 \omega_{x}(f, \delta)
$$

we can immediately write all of the above estimations with $\omega_{x}$ instead of $w_{x}$. Remark 3 . In the case $p=2$ our assumptions confine to the function $\varphi$ only and thus the mentioned result of Xiehua [11, Theorem 3.1, p. 84] follows immediately from Theorem 4, by Remark 2 .

Remark 4. The convergence at Lebesgue's and strong Lebesgue's points were investigated in the papers of Skopina [8] and of Kelly, Kon, Raphael [4]. tions.

We very thank the referee for helpful comments and constructive sugges- 
Open Access. This article is distributed under the terms of the Creative Commons Attribution 4.0 International License (http://creativecommons.org/licenses/ by/4.0/), which permits unrestricted use, distribution, and reproduction in any medium, provided you give appropriate credit to the original author(s) and the source, provide a link to the Creative Commons license, and indicate if changes were made.

\section{References}

[1] Daubechies, I.: Orthonormal bases of compactly supported wavelets. Commun. Pure Appl. Math. XLI, 909-996 (1988)

[2] Daubechies, I.: Ten lectures on wavelets. SIAM, Philadelphia (1992)

[3] DeVore, R.A.: The approximation of continuous functions by positive linear operators. Lecture Notes in Math., vol. 293. Springer, Berlin (1972)

[4] Kelly, S.E., Kon, M.A., Raphael, L.A.: Local convergence for wavelet expansions. J. Funct. Anal. 126(1), 102-138 (1995)

[5] Lal, S., Kumar, M.: Approximation of functions of space $L^{2}(\mathbb{R})$ by wavelet expansions. Lobachevskii J. Math. 34(2), 163-172 (2013)

[6] Meyer, Y.: Ondelettes et opérateurs, vol. 1. Herman, Paris (1990)

[7] Novikov, I.Ya., Protasov, V.Yu., Skopina, M.A.: Wavelet theory. AMS, Providence (2011)

[8] Skopina, M.A.: Local convergence of Fourier series with respect to periodized wavelets. J. Approx. Theory 94, 191-202 (1998)

[9] Skopina, M.A.: Wavelet approximation of periodic functions. J. Approx. Theory 104, 302-329 (2000)

[10] Walter, G.G.: Pointwise convergence of wavelet expansions. J. Approx. Theory 80, 108-118 (1995)

[11] Xiehua, S.: On the degree of approximation by wavelet expansions. Approx. Theory Appl. 14(1), 81-90 (1998)

Włodzimierz Łenski and Bogdan Szal

Faculty of Mathematics

Computer Science and Econometrics

University of Zielona Góra

ul. Szafrana 4a

65-516 Zielona Gora

Poland

e-mail: W.Lenski@wmie.uz.zgora.pl;

B.Szal@wmie.uz.zgora.pl

Received: May 17, 2016.

Accepted: October 11, 2016. 